\title{
An Analytical Framework to Infer Multihop Path Reliability in MANETs
}

\author{
Nelson Antunes, Gonçalo Jacinto and António Pacheco
}

\begin{abstract}
Due to complexity and intractability reasons, most of the analytical studies on the reliability of communication paths in mobile ad hoc networks are based on the assumption of link independence. In this paper, an analytical framework is developed to characterize the random behavior of a multihop path and derive path metrics to characterize the reliability of paths. This is achieved through the modeling of a multihop path as a PDMP (piecewise deterministic Markov process). Two path based metrics are obtained as expectations of functionals of the process: the mean path duration and the path persistence. We show that these metrics are the unique solution of a set of integro-differential equations and provide a recursive scheme for their computation. Finally, numerical results illustrate the computation of the metrics; these results are compared with independent link approximation results.
\end{abstract}

\section{Introduction}

Mobile ad hoc networks (MANETs) nodes can dynamically form a network in a self-organizing manner without the need of an existing fixed infrastructure. An inherent property of these networks is that communication between nodes usually occurs over a multihop path. Therefore, the functionality of the network depends on the reliability of communication paths. On the other

Nelson Antunes

FCT da Universidade do Algarve and CEMAT, e-mail: nantunes@ulag.pt

Gonçalo Jacinto

CIMA and Departmento de Matemática, Universidade de Évora, e-mail: gjcj@uevora.pt

António Pacheco

CEMAT and Departmento of Matemática, Instituto Superior Técnico, Universidade

Técnica de Lisboa e-mail: apacheco@math.ist.utl.pt 
hand, these are dependent on the stability of the links along the path and their dependence structure. In the case of a link failure, the path needs to be either repaired, by finding another link, or replaced with a newly found path. The rerouting operations affect the quality of service and degrade network performance.

Modeling the random movement of nodes in a multihop path plays an important role in examining the statistical properties of link and path reliability. In the general case, the evolution of a multihop path in an ad hoc network, requires a systematic formulation of the geometrical relations governing the complex problem of random movement of the nodes along the multihop path, with the state of its links limited by power constraints and channel effects. A link shares a common node with each of its neighboor links. These dependences on the mobility of the shared nodes between links, may be extended to other links in the case of group mobility models where the mobility of nodes is correlated. The multihop path model should be used to predict the evolution of a path through the derivation of path metrics characterizing its reliability. These metrics can then be used in the design and performance evaluation of routing strategies. Given that a path is set-up (or already active) at time 0 , the path duration refers to the amount of time the path remains available until one of its links fails for the first time. The path persistence is defined as the probability that the path is continuously in existence until time $t_{0}$ provided the path is set-up (or already active) at time 0 .

This paper proposes an analytical framework to fully describe the random behavior of a multihop path in ad hoc networks and obtain path based metrics for computing its reliability. The path is characterized through a Picewise Deterministic Markov Processes (PDMP, see Davis [3]) where, for simplicity, the mobility of each node along the path is given by the random walk model (other models [2], including group mobility models, admit a PDMP description of a multihop path; this fact will be explored in future work). A PDMP is a Markov process that follows deterministic trajectories between random jumps, which occur either spontaneously, in a Poisson-like fashion, or when the process hits the boundary of its state space. We consider two main expectations of functionals of the multihop process: the mean path duration and the path persistence for different initial conditions. We establish that these path metrics are the unique solution of a set of integro-differential equations. Since direct methods to solve them are problematic, we introduce a recursive method by which numerical solutions of the metrics can be obtained. Our work creatively applies the power of the PDMP formalism and the methods presented in [3] (Chapters 2 and 3) to study the reliability of multihops paths in ad hoc networks. Finally, we compute numerical results for the metrics and compare them with those obtained when assuming independent links.

An exact analysis of the reliability of multihop paths appears to be unavailable in the literature. Most analytical studies that focus on link stability extend the analysis for multihop paths assuming independent link failures (e.g., $[4,7,8,9])$. In $[7]$, the path availability has been analyzed when nodes 
move according to the random walk mobility model. [8] investigates the path duration assuming that nodes do not change direction. Xu et al. [9] used a Markov chain mobility model to derive several path metrics. Han et al. [4] proved that when the link count is large, the distribution of path duration converges to an exponential distribution. La and Han [6] relax the independence assumption for the links in [4] requiring that the dependence between links goes away asymptotically with increasing link count. Bai et al. [1] investigate path durations under different mobility models and routing protocols based on simulations.

The paper is organized as follows. Section 2 describes the multihop path model. Section 3 shows that the mean path duration and the path persistence are the unique solution of a set of integro-differential equations. Section 4 gives a recursive method to apply in the computation of the path metrics. Numerical results are presented in Section 5. Finally, Section 6 concludes the paper and gives some research directions.

\section{Multihop Path}

We consider that a multihop path is set-up (or already active) at time 0 with $N-1$ links and extends from node 1 along nodes $2,3, \ldots$, until it reaches node $N$. Each node in the path moves across the plane independently of other nodes according to a variation of the random walk mobility model [2] next described.

\subsection{Random Walk Mobility Model}

A node alternates between two phases: pause (0) and move (1). If at a transition instant a node goes into phase $i$, the amount of time it stays in phase $i$ is drawn independently of the past according to a continuous distribution function $F_{i}$ with support on the set $\mathbb{R}^{+}$. We assume that the hazard rate function of $F_{i}$, denoted by $\lambda_{i}(t)=\mathrm{d} F_{i}(t) /\left(1-F_{i}(t)\right)$, is bounded on the positive reals. When the phase of a node changes to move, the node picks a mobility vector according to a distribution function $F_{M}$ on an open set $S_{M}$. Choosing a mobility vector $m$ corresponds to choosing independently a direction $\theta$ and a velocity $v$ through $m=(v \cos \theta, v \sin \theta)$. The node travels from the current location in the direction and with the velocity drawn from the mobility vector during the entire phase duration, with distribution $F_{1}$. Once this time expires, independently of the past, the node pauses for a random time period with distribution $F_{0}$ before starting to move again. 


\subsection{Link Duration}

We consider a transmission range $R$ equal for all nodes in the multihop path. Given two consecutive nodes in the path, $i-1$ and $i$ with locations in the plane $l^{i-1}$ and $l^{i}$, respectively, they can communicate if $\left\|l^{i-1}-l^{i}\right\|<R$.

In cellular networks, the characterization of the handoff metrics is based on the analysis of the movement of a node with respect to a fixed base station [5]. In ad hoc networks, the link duration can be transformed into the handoff problem by considering the relative movement between the two link nodes. Let $p^{j}$ denote the phase of node $j$ and $m^{j}$ its mobility vector if $p^{j}=1$ (i.e the node is in the move phase). The relative location and relative mobility vector of node $i$ with respect to node $i-1$ are defined, respectively, by

$$
l_{r}^{i}=l^{i}-l^{i-1}, \quad m_{r}^{i}=m^{i}-m^{i-1}
$$

where $m^{i}$ (resp. $m^{i-1}$ ) is omitted in the expression if $p^{i}=0$ (resp. $p^{i-1}=$ $0)$, and if both nodes are in pause phases $m_{r}^{i}=\mathbf{0}$ with $\mathbf{0}=(0,0)$. Let $x \rightarrow(\|x\|, \theta(x))$ denote the one-to-one correspondence between the cartesian coordinates in the plane and the polar coordinates on $\{\mathbf{0}\} \cup \mathbb{R}^{+} \times[0,2 \pi[$ with $\mathbf{0}$ being the polar coordinates of the cartesian origin. From the relative mobility vector $m_{r}^{i}$, the direction and velocity of node $i$ with respect to node $i-1$ are, respectively, $\theta\left(m_{r}^{i}\right)$ and $\left\|m_{r}^{i}\right\|$. In Figure ??, it is shown the relative motion of node $i$, within the transmission region $S_{L}=\left\{x \in \mathbb{R}^{2}:\|x\|<R\right\}$ with respect to node $i-1$. After traveling a distance

$$
Z\left(l_{r}^{i}, m_{r}^{i}\right)=\sqrt{R^{2}-\left(\left\|l_{r}^{i}\right\| \sin \theta^{\prime}\right)^{2}}-\left\|l_{r}^{i}\right\| \cos \theta^{\prime}
$$

where $\theta^{\prime}=\left|\theta\left(m_{r}^{i}\right)-\theta\left(l_{r}^{i}\right)\right|$, node $i$ moves out of the range of node $i-1$. The duration of the link $i$ is

$$
d_{l i n k}\left(l_{r}^{i}, m_{r}^{i}\right)=Z\left(l_{r}^{i}, m_{r}^{i}\right) /\left\|m_{r}^{i}\right\|
$$

for $m_{r}^{i} \neq \mathbf{0}$. Case $m_{r}^{i}=\mathbf{0}$, the duration of the link is infinity and we set $d_{\text {link }}\left(l_{r}^{i}, m_{r}^{i}\right)=\infty$.

\subsection{Multihop path model}

To characterize the multihop path as a PDMP we need to incorporate for each node in the path, 'the phase', 'the elapsed time since last phase transition', 'the mobility vector' and 'the relative location with respect to the previous node'. Thus, we obtain a process $\mathbf{X}=(\mathbf{P}, \mathbf{A})$ with $\mathbf{P}=\left(P^{i}\right)_{1 \leq i \leq N}$ where $P^{i}$ is the phase process of node $i$, and $\mathbf{A}=\left(\mathbf{E}, \mathbf{M}, \mathbf{L}_{r}\right)$ where $\mathbf{E}=\left(E^{i}\right)_{1 \leq i \leq N}$, $\mathbf{M}=\left(M^{i}\right)_{1 \leq i \leq N}$ and $\mathbf{L}_{r}=\left(L_{r}^{i}\right)_{2 \leq i \leq N}$. The process $E^{i}$ gives the elapsed time since the last phase transition of node $i, M^{i}$ is the mobility vector process of 
node $i$ if $P^{i}(t)=1$ (and is omitted if $P^{i}(t)=0$ ), and finally, $L_{r}^{i}$ is the process of the relative location of node $i$ with respect to node $i-1$. The process $\mathbf{X}$ is a PDMP characterized as follows.

\section{The Deterministic Motion and State Space}

Constructing a PDMP as a path model requires a description of the deterministic trajectory of the process between random jumps along with the explicit definition of the boundary of its state space where jumps occur.

From the definition of $\mathbf{X}$, a state will be denoted by $\mathbf{x}=(\mathbf{p}, \mathbf{a})$ where $\mathbf{a}=$ $\left(\mathbf{e}, \mathbf{m}, \mathbf{l}_{r}\right)$ with the vector $\mathbf{p}=\left(p^{1}, \ldots, p^{N}\right)$ containing the phases of nodes, $\mathbf{e}=\left(e^{1}, \ldots, e^{N}\right)$ the elapsed times of the nodes in their current phases, $\mathbf{m}=$ $\left(m^{1}, \ldots, m^{N}\right)$ including the mobility vectors of the nodes, having dimension $N$ when all $p^{j}=1$ and with $m^{j}$ omitted if $p^{j}=0$, and $\mathbf{l}_{r}=\left(l_{r}^{2}, \ldots, l_{r}^{N}\right)$ the relative locations of nodes $2,3, \ldots, N$ relative to nodes $1,2, \ldots, N-1$, respectively. From a state $\mathbf{x}$, the deterministic trajectory of $\mathbf{X}$ until the next jump is characterized by $\phi(t, \mathbf{x})=\left(\mathbf{p}, \phi_{\mathbf{p}}(t, \mathbf{a})\right)$ with

$$
\phi_{\mathbf{p}}(t, \mathbf{a})=\left(\mathbf{e}+t \mathbf{1}, \mathbf{m}, \mathbf{l}_{r}+t \mathbf{m}_{r}\right), \quad t \in \mathbb{R}
$$

representing the evolution of the component a over time, where $\mathbf{1}$ denotes a vector of $1^{\prime} s$ with dimension $N$ and the vector $\mathbf{m}_{r}=\left(m_{r}^{2}, \ldots, m_{r}^{N}\right)$ contains the relative mobility vectors of nodes $2,3, \ldots, N$ relative to nodes $1,2, \ldots, N-1$, respectively.

Consider the set

$$
\left.S_{\mathbf{p}}=\right] 0, \infty\left[{ }^{N} \times S_{M} \sum p_{i} \times S_{L}^{N-1}\right.
$$

where we recall that $S_{M}$ and $S_{L}$ are open sets, and let $\partial S_{\mathbf{p}}$ denote the boundary of the set $S_{\mathbf{p}}$. Now, define

$$
\partial^{+} S_{\mathbf{p}}=\left\{\mathbf{a}^{\prime} \in \partial S_{\mathbf{p}}: \mathbf{a}^{\prime}=\phi_{\mathbf{p}}(t, \mathbf{a}) \text { for some } \mathbf{a} \in S_{\mathbf{p}}, t>0\right\}
$$

given by the set of boundary points at which the multihop path process exits from $S_{\mathbf{p}}$ and

$$
\partial^{-} S_{\mathbf{p}}=\left\{\mathbf{a}^{\prime} \in \partial S_{\mathbf{p}}: \mathbf{a}^{\prime}=\phi_{\mathbf{p}}(-t, \mathbf{a}) \text { for some } \mathbf{a} \in S_{\mathbf{p}}, t>0\right\}
$$

the set of boundary points that take the process into $S_{\mathbf{p}}$. The disjoint union of the sets $\partial^{+} S_{\mathbf{p}}$ is

$$
B=\coprod_{\mathbf{p} \in\{0,1\}^{n}} \partial^{+} S_{\mathbf{p}}=\left\{(\mathbf{p}, \mathbf{a}): \mathbf{p} \in\{0,1\}^{n}, \mathbf{a} \in \partial^{+} S_{\mathbf{p}}\right\}
$$


which represents the set of states through which the multihop path disconnects. When the process hits a state in the boundary $B$, it means that the path breaks and $\mathbf{X}$ jumps to an absorbing state which we denote by $\Delta$. Therefore, the state space of $\mathbf{X}$ becomes $S_{\mathbf{X}}^{\Delta}=S_{\mathbf{X}} \cup\{\Delta\}$, where $S_{\mathbf{X}}$ denotes the disjoint union of the sets $S_{\mathbf{p}}^{-}=S_{\mathbf{p}} \cup \partial^{-} S_{\mathbf{p}}$,

$$
S_{\mathbf{X}}=\coprod_{\mathbf{p} \in\{0,1\}^{n}} \mathbf{S}_{\mathbf{p}}^{-}=\left\{(\mathbf{p}, \mathbf{a}): \mathbf{p} \in\{0,1\}^{n}, \mathbf{a} \in S_{\mathbf{p}}^{-}\right\}
$$

For $\mathbf{x} \in S_{\mathbf{X}}$, define $d_{\text {path }}(\mathbf{x})$ as the path duration (i.e. the time to hit a state in $B$ ) constrained to no phase transitions of the nodes taking place when starting from state $\mathbf{x}$,

$$
\begin{aligned}
d_{\text {path }}(\mathbf{x}) & =\inf \{t>0: \phi(t, \mathbf{x}) \in B\} \\
& =\inf \left\{d_{l i n k}\left(l_{r}^{i}, m_{r}^{i}\right): i=2, \ldots, N\right\} .
\end{aligned}
$$

This time is equal to infinity if all nodes are in pause phase or all nodes have the same mobility vector.

\section{The Jump Rate}

The function $\boldsymbol{\lambda}: S_{\mathbf{X}}^{\Delta} \rightarrow \mathbb{R}_{0}^{+}$characterizes the jump rate in each state of the process. For $\mathbf{x} \in S_{\mathbf{X}}$ the jump rate depends only on the phase and the time since last phase transition of each node, and is given by the sum of the hazard rate functions $\lambda_{i}(t)=\mathrm{d} F_{i}(t) /\left(1-F_{i}(t)\right)$ of the phase duration distributions $F_{i}$,

$$
\boldsymbol{\lambda}(\mathbf{x})=\sum_{i=1}^{n} \lambda_{p^{i}}\left(e^{i}\right)
$$

while at the absorbing state $\boldsymbol{\lambda}(\Delta)=0$.

\section{The Transition Measure}

Before introducing its definition, first a note about notation. For a vector $\mathbf{y}=\left(y^{1}, \ldots, y^{n}\right)$, let $\mathbf{y}^{\prime}=[\mathbf{y}]_{z}^{j}$ denote a vector that differs from $\mathbf{y}$ only on the component $j$, taking the value $z$ on that component (i.e., $\mathbf{y}^{\prime}$ is given by $y^{\prime j}=z$ and $\left.y^{\prime i}=y^{i}, i \neq j\right)$. In addition, we let $\mathbf{y}_{\backslash j}$ denote the vector obtained from $\mathbf{y}$ by omitting $y^{j}$ and retaining $y^{i}$ for all $i \neq j$. The interpretation of $\mathbf{P}$ and $\mathbf{A}$ makes it clear that from any $\mathbf{x}=(\mathbf{p}, \mathbf{a}) \in S_{\mathbf{X}}$ it is only possible to jump to a state where a node changes its phase characteristics (phase, elapsed time in the phase, and mobility vector) and all the other values of the components remain the same, i.e. for some $j$, we make the transition 


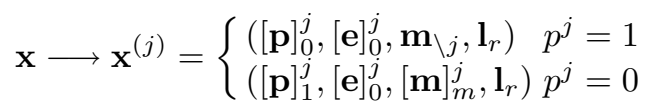

with $m \in S_{M}$. The transition measure $Q:\left(S_{\mathbf{X}} \cup B\right) \times \mathcal{E} \rightarrow[0,1]$, with $\mathcal{E}$ denoting the event space of $S_{\mathbf{X}}$, is such that for $\mathbf{x} \in S_{\mathbf{X}}, Q(\mathbf{x}, \cdot)$ is a probability measure defined by

$$
\begin{array}{ll}
Q\left(\mathbf{x},\left\{\mathbf{x}^{(j)}\right\}\right)=\lambda_{p^{j}}\left(e^{j}\right) / \boldsymbol{\lambda}(\mathbf{x}) & p^{j}=1 \\
Q\left(\mathbf{x}, \mathbf{d} \mathbf{x}^{(j)}\right)=\lambda_{p^{j}}\left(e^{j}\right) / \boldsymbol{\lambda}(\mathbf{x}) F_{M}(\mathrm{~d} m) & p^{j}=0
\end{array}
$$

and for $\mathbf{x} \in B$ we have $Q(\mathbf{x},\{\Delta\})=1$.

\section{The Motion of the Multihop Path Process}

Putting all the above together, the evolution of $\mathbf{X}$ starting from state $\mathbf{x} \in S_{\mathbf{X}}$ can be constructed as follows. The survivor function of the first jump time $T_{1}$ is defined by

$$
G_{\mathbf{x}}(t)=\left\{\begin{array}{lr}
\exp \left(-\int_{0}^{t} \boldsymbol{\lambda}(\phi(s, \mathbf{x})) \mathrm{d} s\right) & t<d_{\text {path }}(\mathbf{x}) \\
0 & t \geq d_{\text {path }}(\mathbf{x})
\end{array}\right.
$$

and the state at an instant of time before the first jump is

$$
\mathbf{X}(t)=\phi(t, \mathbf{x}), \quad t<T_{1} .
$$

If $T_{1}<d_{\text {path }}(\mathbf{x})$ one of the nodes in the path changes phase and the next state of the multihop process $\mathbf{X}\left(T_{1}\right)$ has distribution $Q\left(\phi\left(T_{1}, \mathbf{x}\right), \cdot\right)$ given by (1). Otherwise, $T_{1}=d_{\text {path }}(\mathbf{x})$ which means that the path breaks since the process hits a state in $B$ and the next state $\mathbf{X}\left(T_{1}\right)$ is $\Delta$ with probability 1; the process then stays in $\Delta$ forever since the jump rate out of $\Delta$ is zero. The process restarts from $\mathbf{X}\left(T_{1}\right)$ in a similar way if $T_{1}<d_{\text {path }}(\mathbf{x})$ with survivor function of the next inter jump time $T_{2}-T_{1}$ given by $G_{\mathbf{X}\left(T_{1}\right)}$ and so on ....

\section{Remark}

An inspection in the definition of the PDMP shows that technical problems will arise if mobility vectors can assume values from a closed set $S_{M}$. We can relax this assumption when the velocities or directions that describe the mobility vectors are discrete sets. This includes the case of one dimensional ad hoc network with directions on a straight line. All that needs to be done is to consider the discrete quantities (velocities or directions) in the first component of $\mathbf{X}$, along with the node phases, and to redefine the PDMP properly according to these changes. In the rest of the paper, we shall focus 
only on the assumptions defined in Subsection 2.1 but similar results can be derived.

\subsection{Multihop path set up}

We assume that at time $t=0$ the state of a multihop path with $N$ nodes is drawn according to some specified initial distribution (see Section 5 for an initialization rule).

\section{Path based metrics}

In this section we derive two of the main path metrics to characterize the reliability of a multihop path as the expectation of a functional of the process $\mathrm{X}$.

\subsection{Mean path duration}

Given the state of the multihop path process $\mathbf{x} \in S_{\mathbf{X}}^{\Delta}$, the mean path duration is denoted by

$$
D(\mathbf{x})=\mathbb{E}_{\mathbf{x}}\left(\int_{0}^{\infty} \mathbf{I}_{S \mathbf{X}}(\mathbf{X}(s)) \mathrm{d} s\right)
$$

where $\mathbf{I}_{A}$ is the indicator function of a set $A$. Let $f: S \Delta \cup B \rightarrow \mathbb{R}^{+}$be a bounded measurable function and for $\mathbf{x} \in B$ define $f(\mathbf{x}) \equiv \lim _{t \downarrow 0} f(\phi(-t, \mathbf{x}))$. Let $\mathcal{Q}$ be an operator mapping the set of bounded measurable functions $S_{\mathbf{X}} \cup$ $B$ into itself such that $\mathcal{Q} f$ is a function defined by $\mathcal{Q} f(\mathbf{x})=\int_{S_{\mathbf{x}}} f(\mathbf{y}) Q(\mathbf{x}, \mathbf{d y})$ and therefore for $\mathbf{x} \in S_{\mathbf{X}}$,

$$
\begin{aligned}
\mathcal{Q} f(\mathbf{x})=\sum_{\left\{j: p^{j}=0\right\}} & \int_{\left\{m: m \in S_{M}\right\}} Q\left(\mathbf{x}, \mathbf{d} \mathbf{x}^{(j)}\right) f\left(\mathbf{x}^{(j)}\right) \\
& +\sum_{\left\{j: p^{j}=1\right\}} Q\left(\mathbf{x},\left\{\mathbf{x}^{(j)}\right\}\right) f\left(\mathbf{x}^{(j)}\right)
\end{aligned}
$$

and for $\mathbf{x} \in B, \mathcal{Q} f(\mathbf{x})=f(\Delta)$.

For $\mathbf{x} \in S_{\mathbf{X}}$, the state of $\mathbf{X}$ after a short time $t$ is, roughly, $\phi(t, \mathbf{x})$ with probability $(1-\boldsymbol{\lambda}(\mathbf{x}) t)$, while with probability $\boldsymbol{\lambda}(\mathbf{x}) t$ the process jumps to another state $\mathbf{X}(t)$ with transition measure $Q$ and all other events have probability $o(t)$. Thus, we have 


$$
\mathbb{E}_{\mathbf{x}}(f(\mathbf{X}(t)))=(1-\boldsymbol{\lambda}(\mathbf{x}) t) f(\phi(t, \mathbf{x}))+\boldsymbol{\lambda}(\mathbf{x}) t \mathcal{Q} f(\phi(t, \mathbf{x}))+o(t)
$$

so that

$$
\begin{aligned}
\frac{1}{t} \mathbb{E}_{\mathbf{x}}(f(\mathbf{X}(t)) & -f(\mathbf{x}))=\frac{1}{t}(f(\phi(t, \mathbf{x}))-f(\mathbf{x})) \\
& +\boldsymbol{\lambda}(\mathbf{x})(\mathcal{Q} f(\phi(t, \mathbf{x}))-f(\phi(t, \mathbf{x})))+o(1) .
\end{aligned}
$$

Let $\mathcal{A}$ denote an operator acting on the domain of bounded measurable realvalued functions on $S_{\mathbf{X}}^{\Delta} \cup B$ defined by

$$
\mathcal{A} f(\mathbf{x})=\lim _{t \rightarrow 0} \frac{1}{t} \mathbb{E}_{\mathbf{x}}(f(\mathbf{X}(t))-f(\mathbf{x})) .
$$

In order to define the derivative of $f$ with respect to the flow $\phi(t, \mathbf{x})$ in a rigorous way, we need to define its phase function by $f_{\mathbf{p}}: S_{\mathbf{p}}^{-} \rightarrow \mathbb{R}^{+}$such that $f_{\mathbf{p}}(\mathbf{a})=f(\mathbf{x})$. If for a state $\mathbf{x}$ the function $t \rightarrow f_{\mathbf{p}}\left(\phi_{\mathbf{p}}(t, \mathbf{a})\right)$ is differentiable almost everywhere on $\left[0, d_{\text {path }}(\mathbf{x})[\right.$ then

$$
\frac{\mathrm{d}}{\mathrm{d} t} f_{\mathbf{p}}\left(\phi_{\mathbf{p}}(t, \mathbf{a})\right)=\mathcal{V}_{\mathbf{p}} f_{\mathbf{p}}\left(\phi_{\mathbf{p}}(t, \mathbf{a})\right), \quad \phi_{\mathbf{p}}(0, \mathbf{a})=\mathbf{a}
$$

holds for almost all $t$, and where $\mathcal{V}_{\mathbf{p}}$ is a vector field and $\phi_{\mathbf{p}}(t, \mathbf{a})$ is the unique integral curve of $\mathcal{V}_{\mathbf{p}}$ such that (5) is satisfied.

Therefore, as $t \rightarrow 0$ in equation (4) we obtain

$$
\mathcal{A} f(\mathbf{x})=\mathcal{V} f(\mathbf{x})+\boldsymbol{\lambda}(\mathbf{x})(\mathcal{Q} f(\mathbf{x})-f(\mathbf{x})),
$$

for $\mathbf{x} \in S_{\mathbf{X}}$, where to simplify the notation we write $\mathcal{V} f(\mathbf{x})$ instead of the more accurate $\mathcal{V}_{\mathbf{p}} f_{\mathbf{p}}(\mathbf{a})$. Also any reference to a function $t \rightarrow f(\phi(t, \mathbf{x}))$ should be read as $t \rightarrow f_{\mathbf{p}}\left(\phi_{\mathbf{p}}(t, \mathbf{a})\right)$.

The next result follows from Theorems 32.2 and 32.10 of Davis [3] conveniently applied to the expectation functional $D$ of the PDMP $\mathbf{X}$ with finite time horizon and taking into account the specific boundary conditions.

Proposition 1. For each $\mathbf{x} \in S_{\mathbf{X}}, t \rightarrow D(\phi(t, \mathbf{x}))$ is absolutely continuous on $\left[0, d_{\text {path }}(\mathbf{x})\right.$ [ and $D$ is the unique bounded solution of the equations

$$
\mathcal{A} f(\mathbf{x})=-1, \quad \mathbf{x} \in S_{\mathbf{X}},
$$

and at a boundary state $\mathbf{x} \in B, f(\mathbf{x})=f(\Delta)=0$.

The result above states that for $\mathbf{x} \in S_{\mathbf{X}}$ the function $t \rightarrow D(\phi(t, \mathbf{x}))$ is differentiable almost everywhere on $\left[0, d_{\text {path }}(\mathbf{x})\right.$ [ with derivative denoted by $\mathcal{V} D\left(\mathbf{x}^{\prime}\right)$ at $\mathbf{x}^{\prime}=\phi(t, \mathbf{x})$, when it exists. The value of the derivative at such points is equal to

$$
-1-\boldsymbol{\lambda}\left(\mathbf{x}^{\prime}\right)\left(\mathcal{Q} D\left(\mathbf{x}^{\prime}\right)-D\left(\mathbf{x}^{\prime}\right)\right) .
$$


Because (6) includes an integral term, the equations are systems of integrodifferential equations. Solving them provides a way of computing the mean path duration for different initial conditions.

Proof. By definition $D(\Delta)=0$. We assume that $\mathbf{x} \in S_{\mathbf{X}}$ and $\left.t \in\right] 0, d_{\text {path }}(\mathbf{x})[$. By the strong Markov property, the function $D$ satisfies

$$
\begin{aligned}
D(\mathbf{x})= & \mathbb{E}_{\mathbf{x}}\left(\left(T_{1} \wedge t\right)+D\left(\phi\left(T_{1} \wedge t, \mathbf{x}\right)\right)\right) \\
= & G_{\mathbf{x}}(t)(t+D(\phi(t, \mathbf{x}))) \\
& +\mathbb{E}_{\mathbf{x}}\left(\left(T_{1}+\mathcal{Q} D\left(\phi\left(T_{1}, \mathbf{x}\right)\right)\right) \mathbf{I}_{0, t]}\left(T_{1}\right)\right) .
\end{aligned}
$$

Using the density function of first phase transition time $T_{1}$ on $[0, t]$, given by $\lambda(\phi(s, \mathbf{x})) G_{\mathbf{x}}(s)$, the second term on the right hand side of $(7)$ is

$$
\begin{aligned}
& \mathbb{E}_{\mathbf{x}}\left(\left(T_{1}+\mathcal{Q} D\left(\phi\left(T_{1}, \mathbf{x}\right)\right)\right) \mathbf{I}_{] 0, t]}\left(T_{1}\right)\right) \\
& =\int_{0}^{t} \lambda(\phi(s, \mathbf{x})) G_{\mathbf{x}}(s)\left(\int_{0}^{s} 1 \mathrm{~d} v+\mathcal{Q} D(\phi(s, \mathbf{x}))\right) \mathrm{d} s \\
& =\int_{0}^{t} G_{\mathbf{x}}(s)(1+\lambda(\phi(s, \mathbf{x})) \mathcal{Q} D(\phi(s, \mathbf{x}))) \mathrm{d} s-G_{\mathbf{x}}(t) t .
\end{aligned}
$$

where the last equality is obtained by changing the order of integration. Then (7) becomes

$$
\begin{aligned}
& D(\phi(t, \mathbf{x}))=G_{\mathbf{x}}(t)^{-1}(D(\mathbf{x}) \\
& \left.-\int_{0}^{t} G_{\mathbf{x}}(s)(1+\lambda(\phi(s, \mathbf{x})) \mathcal{Q} D(\phi(s, \mathbf{x}))) \mathrm{d} s\right) \\
& =G_{\mathbf{x}}(t)^{-1} D(\mathbf{x})-\int_{0}^{t}(1+\lambda(\phi(s, \mathbf{x})) \mathcal{Q} D(\phi(s, \mathbf{x}))) \mathrm{d} s \\
& \quad-\int_{0}^{t} G_{\mathbf{x}}(s)(1+\lambda(\phi(s, \mathbf{x})) \mathcal{Q} D(\phi(s, \mathbf{x}))) \\
& \quad \times\left(G_{\mathbf{x}}(t)^{-1}-G_{\mathbf{x}}(s)^{-1}\right) \mathrm{d} s
\end{aligned}
$$

and since

$$
\int_{s}^{t} \lambda(\phi(v, \mathbf{x})) G_{\mathbf{x}}(v)^{-1} \mathrm{~d} v=G_{\mathbf{x}}(t)^{-1}-G_{\mathbf{x}}(s)^{-1}, 0 \leq s \leq t,
$$

we have 


$$
\begin{aligned}
& D(\phi(t, \mathbf{x}))=\left(\int_{0}^{t} \lambda(\phi(s, \mathbf{x})) G_{\mathbf{x}}(s)^{-1} \mathrm{~d} s+1\right) D(\mathbf{x}) \\
& \quad-\int_{0}^{t}(1+\lambda(\phi(s, \mathbf{x})) \mathcal{Q} D(\phi(s, \mathbf{x}))) \mathrm{d} s \\
& \quad-\int_{0}^{t} G_{\mathbf{x}}(s)(1+\lambda(\phi(s, \mathbf{x})) \mathcal{Q} D(\phi(s, \mathbf{x}))) \\
& \quad \times \int_{s}^{t} \lambda(\phi(v, \mathbf{x})) G_{\mathbf{x}}(v)^{-1} \mathrm{~d} v \mathrm{~d} s \\
& =D(\mathbf{x})-\int_{0}^{t}(1+\lambda(\phi(s, \mathbf{x})) \mathcal{Q} D(\phi(s, \mathbf{x}))) \mathrm{d} s \\
& \quad+\int_{0}^{t} \lambda(\phi(v, \mathbf{x})) G_{\mathbf{x}}(v)^{-1} \\
& \quad \times\left(D(\mathbf{x})-\int_{0}^{v} G_{\mathbf{x}}(s)(1+\lambda(\phi(s, \mathbf{x})) \mathcal{Q} D(\phi(s, \mathbf{x}))) \mathrm{d} s\right) \mathrm{d} v .
\end{aligned}
$$

Thus,

$$
D(\phi(t, \mathbf{x}))=D(\mathbf{x})+\int_{0}^{t} g(s) \mathrm{d} s
$$

where

$$
g(s)=-1-\boldsymbol{\lambda}(\phi(s, \mathbf{x}))(\mathcal{Q} D(\phi(s, \mathbf{x}))-D(\phi(s, \mathbf{x}))) .
$$

In the appendix we prove an auxiliary result which states that $D$ is a bounded function for all $\mathbf{x} \in S_{\mathbf{X}}^{\Delta}$, and $\mathbb{E}_{\mathbf{x}}(D(\mathbf{X}(t)))$ goes to zero as $t$ tends to infinity. Then $\mathcal{Q} D$ is also bounded and $\int_{0}^{t}|g(s)| \mathrm{d} s$ is finite. Therefore, by the fundamental theorem of calculus $t \rightarrow D(\phi(t, \mathbf{x}))$ is absolutely continuous on $[0, t]$ with derivative $g(t)$ and equation (6) is satisfied. Now, let $\mathbf{x}^{\prime} \in B$ and $t>0$ such that the initial state is $\mathbf{x}=\phi\left(-t, \mathbf{x}^{\prime}\right)$. By the strong Markov property, we have

$$
\begin{aligned}
D(\mathbf{x})= & G_{\mathbf{x}}(t)\left(t+\mathcal{Q} D\left(\mathbf{x}^{\prime}\right)\right) \\
& +\int_{0}^{t} \boldsymbol{\lambda}(\phi(s, \mathbf{x})) G_{\mathbf{x}}(s)(1+\mathcal{Q} D(\phi(s, \mathbf{x}))) \mathrm{d} s .
\end{aligned}
$$

Thus, as $t \downarrow 0$ the function $D$ possesses a limit as a boundary state is approached and $D\left(\mathbf{x}^{\prime}\right)=0$.

Under the conditions that for $\mathbf{x} \in S_{\mathbf{X}}, t \rightarrow f(\phi(t, \mathbf{x}))$ is an absolutely continuous function on $\left[0, d_{\text {path }}(\mathbf{x})\right.$ [ and $f$ is a bounded function, from [Davis [3], Theorem 31.3, p. 83] conveniently applied, the process $\left(M^{f}(t)\right)$ defined by

$$
M^{f}(t)=f(\mathbf{X}(t))-f(\mathbf{X}(0))-\int_{0}^{t} \mathcal{A} f(\mathbf{X}(s)) \mathrm{d} s, \quad t \geq 0
$$


is a martingale and $\mathcal{A} f(\mathbf{X}(s))=\lim _{t \rightarrow 0} \frac{1}{t} \mathbb{E}_{\mathbf{X}(s)}(f(\mathbf{X}(s+t))-f(\mathbf{X}(s))$. Since $\left(M^{f}(t)\right)$ is a martingale then taking conditional expectation we have $\mathbb{E}_{\mathbf{x}}\left(M^{f}(t)\right)=M^{f}(0)=0$, which implies that the Dynkin formula holds in the form

$$
f(\mathbf{x})=\mathbb{E}_{\mathbf{x}}(f(\mathbf{X}(t)))-\mathbb{E}_{\mathbf{x}}\left(\int_{0}^{t} \mathcal{A} f(\mathbf{X}(s)) \mathrm{d} s\right) .
$$

In view of (6) and $f(\Delta)=0$, we have that $\mathcal{A} f(\mathbf{X}(s))=-\mathbf{I}_{S_{\mathbf{X}}}(\mathbf{X}(s))$; therefore, if $\lim _{\mathbf{t} \rightarrow \infty} \mathbb{E}_{\mathbf{x}}(f(\mathbf{X}(t)))=0$, by monotone converge theorem we get

$$
f(\mathbf{x})=\mathbb{E}_{\mathbf{x}}\left(\int_{0}^{\infty} \mathbf{I}_{S \mathbf{X}}(\mathbf{X}(s)) \mathrm{d} s\right)
$$

and the solution is unique.

\subsection{Path persistence}

We can derive the probability that path is continuously in existence until time $\left.\left.t_{0} \in\right] 0, t^{*}\right]$ (where $t^{*}>0$ is fixed) provided that the path is alive at time 0 as the expectation of a functional of $\mathbf{X}$. For that, we need to include explicitely the time variation in the state of the PDMP $\mathbf{X}$. We denote the extended multihop path process by $\widehat{\mathbf{X}}$ with state space $S_{\widehat{\mathbf{X}}}^{\Delta}=S_{\widehat{\mathbf{X}}} \cup\{\Delta\}$ where

$$
\left.\left.S_{\widehat{\mathbf{X}}}=S_{\mathbf{X}} \times\right] 0, t^{*}\right]
$$

and the set of the boundary states is represented by

$$
\widehat{B}=\left(B \times\left[0, t^{*}\right]\right) \cup \widehat{B}^{0}
$$

with $\widehat{B}^{0}=S_{\mathbf{X}} \times\{0\}$. From the definition of $\widehat{\mathbf{X}}$, a state in $S_{\widehat{\mathbf{X}}} \cup \widehat{B}$ is denoted by $\widehat{\mathbf{x}}=\left(\mathbf{x}, t_{0}\right)$ where $t_{0}$ is the time variation component and $\mathbf{x}=(\mathbf{p}, \mathbf{a})$ is defined as before. The deterministic evolution of the process from a state $\widehat{\mathbf{x}}$ is given by $\widehat{\phi}(t, \widehat{\mathbf{x}})=\left(\mathbf{p}, \widehat{\phi}_{\mathbf{p}}\left(t,\left(\mathbf{a}, t_{0}\right)\right)\right)$ with

$$
\widehat{\phi}_{\mathbf{p}}\left(t,\left(\mathbf{a}, t_{0}\right)\right)=\left(\phi_{\mathbf{p}}(t, \mathbf{a}), t_{0}-t\right), \quad t \in \mathbb{R} .
$$

Let $\widehat{d}_{\text {path }}(\widehat{\mathbf{x}})$ be the time to hit a state in $\widehat{B}$ constrained to no phase transition of nodes in path when starting from state $\widehat{\mathbf{x}}$,

$$
\begin{aligned}
& \widehat{d}_{\text {path }}(\widehat{\mathbf{x}}) \\
& =\inf \left\{t>0: \widehat{\phi}_{\mathbf{p}}\left(t,\left(\mathbf{a}, t_{0}\right)\right) \in \partial^{+} S_{\mathbf{p}} \times\left[0, t^{*}\right] \cup S_{\mathbf{p}}^{-} \times\{0\}\right\} \\
& =\inf \left\{d_{\text {path }}(\mathbf{x}), t_{0}\right\}
\end{aligned}
$$


When the extended multihop path process hits the boundary $\widehat{B}$, it means that the path breaks or the time variation component has reached zero, and $\widehat{\mathbf{X}}$ jumps to the absorbing state $\Delta$. The remaining parameters of $\widehat{\mathbf{X}}$ are the jump rate $\widehat{\boldsymbol{\lambda}}: S_{\widehat{\mathbf{X}}}^{\Delta} \rightarrow \mathbb{R}^{+}$defined by

$$
\widehat{\boldsymbol{\lambda}}(\widehat{\mathbf{x}})=\boldsymbol{\lambda}(\mathrm{x}), \quad \widehat{\mathbf{x}} \in S_{\widehat{\mathbf{x}}}
$$

and $\widehat{\boldsymbol{\lambda}}(\Delta)=0$; the transition measure $\widehat{Q}:\left(S_{\widehat{\mathbf{x}}} \cup \widehat{B}\right) \times \widehat{\mathcal{E}} \rightarrow[0,1]$, where $\widehat{\mathcal{E}}$ denotes the event space of $S_{\widehat{\mathbf{x}}}$, is such that for $\widehat{\mathbf{x}} \in S_{\widehat{\mathbf{x}}}$ we have

$$
\widehat{Q}\left(\widehat{\mathbf{x}}, \mathbf{d} \mathbf{x}^{(j)} \times\left\{t_{0}\right\}\right)=Q\left(\mathbf{x}, \mathbf{d} \mathbf{x}^{(j)}\right)
$$

where $Q$ is defined in (1) and $\widehat{Q}(\widehat{\mathbf{x}},\{\Delta\})=1$ for $\widehat{\mathbf{x}} \in \widehat{B}$. The extended process $\widehat{\mathbf{X}}$ has the same behavior as $\mathbf{X}$ until it hits a state in boundary $\widehat{B}$. Starting from state $\widehat{\mathbf{x}} \in S_{\widehat{\mathbf{X}}}$, the survivor function of first jump time $\widehat{T}_{1}$ of the process $\widehat{\mathbf{X}}$ is given by

$$
\widehat{G}_{\widehat{\mathbf{x}}}(t)=\mathbf{I}_{\left[0, \widehat{d}_{\mathrm{path}}(\widehat{\mathbf{x}})[\right.}(t) G_{\mathbf{x}}(t), \quad t \geq 0,
$$

and

$$
\widehat{\mathbf{X}}(t)=\widehat{\phi}(t, \widehat{\mathbf{x}}), \quad t<\widehat{T}_{1} .
$$

The next state $\widehat{\mathbf{X}}\left(\widehat{T}_{1}\right)$ has distribution $\widehat{Q}\left(\widehat{\phi}\left(\widehat{T}_{1}, \widehat{\mathbf{x}}\right), \cdot\right)$. If $\widehat{\mathbf{X}}\left(\widehat{T}_{1}\right)=\Delta$ the process stays there forever since the jump rate out of $\Delta$ is zero. Otherwise, the process restarts from $\widehat{\mathbf{X}}\left(\widehat{T}_{1}\right)$ in a similar way if $\widehat{T}_{1}<\widehat{d}_{\text {path }}(\widehat{\mathbf{x}})$ with survivor function of the next inter jump time $\widehat{T}_{2}-\widehat{T}_{1}$ given by $\widehat{G}_{\widehat{\mathbf{X}}\left(\widehat{T}_{1}\right)}$ and so on ....

The path persistence at time $\left.\left.t_{0} \in\right] 0, t^{*}\right]$ starting from $\mathbf{x} \in S_{\mathbf{X}}$ can be written as expectation of a functional of $\widehat{\mathbf{X}}$ by

$$
U\left(\mathbf{x}, t_{0}\right)=\mathbb{E}_{\left(\mathbf{x}, t_{0}\right)}\left(\mathbf{I}_{\widehat{B}^{0}}\left(\widehat{\mathbf{X}}\left(\widehat{T}_{*}^{-}\right)\right)\right)
$$

where $\widehat{T}_{*}=\left\{\widehat{T}_{i}: \widehat{X}\left(\widehat{T}_{i}^{-}\right) \in \widehat{B}\right\}$ is the time to reach the absorbing state $\Delta$ and, by convention, $U(\Delta)=0$.

Let $f: S_{\widehat{\mathbf{x}}}^{\Delta} \cup \widehat{B} \rightarrow \mathbb{R}^{+}$denote any bounded measurable function and at the boundary state $\widehat{\mathbf{x}} \in \widehat{B}$ we define $f(\widehat{\mathbf{x}}) \equiv \lim _{t \downarrow 0} f(\widehat{\phi}(-t, \widehat{\mathbf{x}}))$. Let $\widehat{\mathcal{Q}}$ denote an operator mapping the set of bounded measurable functions on $S_{\widehat{\mathbf{x}}} \cup \widehat{B}$ into itself. The operation of $\widehat{\mathcal{Q}}$ on $f$ is a function defined by $\widehat{\mathcal{Q}} f(\widehat{\mathbf{x}})=$ $\int_{S_{\widehat{\mathbf{x}}}} f(\widehat{\mathbf{y}}) \widehat{Q}(\widehat{\mathbf{x}}, \mathbf{d} \widehat{\mathbf{y}})$ and for $\widehat{\mathbf{x}} \in S_{\widehat{\mathbf{x}}}$ we have

$$
\begin{aligned}
\widehat{\mathcal{Q}} f(\widehat{\mathbf{x}})= & \sum_{\left\{j: p^{j}=0\right\}} \int_{\left\{m: m \in S_{M}\right\}} Q\left(\mathbf{x}, \mathbf{d} \mathbf{x}^{(j)}\right) f\left(\mathbf{x}^{(j)}, t_{0}\right) \\
& +\sum_{\left\{j: p^{j}=1\right\}} Q\left(\mathbf{x},\left\{\mathbf{x}^{(j)}\right\}\right) f\left(\mathbf{x}^{(j)}, t_{0}\right)
\end{aligned}
$$


and for $\widehat{\mathbf{x}} \in \widehat{B}, \widehat{\mathcal{Q}} f(\widehat{\mathbf{x}})=f(\Delta)$. Using the same heuristics arguments as in Subsection 3.1 for $\widehat{\mathbf{x}} \in S_{\widehat{\mathbf{x}}}$ we obtain

$$
\begin{aligned}
\frac{1}{t} \mathbb{E}_{\widehat{\mathbf{x}}}(f(\widehat{\mathbf{X}}(t))-f(\widehat{\mathbf{x}}))=\frac{1}{t}(f(\widehat{\phi}(t, \widehat{\mathbf{x}}))-f(\widehat{\mathbf{x}})) \\
+\boldsymbol{\lambda}(\mathbf{x})(\widehat{\mathcal{Q}} f(\widehat{\phi}(t, \widehat{\mathbf{x}}))-f(\widehat{\phi}(t, \widehat{\mathbf{x}})))+o(1) .
\end{aligned}
$$

In addition, let $\widehat{\mathcal{A}}$ be an operator acting on the domain of bounded measurable real-valued functions on $S_{\widehat{\mathbf{x}}} \cup \widehat{B}$ such that $\widehat{\mathcal{A}} f$ is a function defined by

$$
\widehat{\mathcal{A}} f(\widehat{\mathbf{x}}) \equiv \lim _{t \rightarrow 0} \frac{1}{t} \mathbb{E}_{\widehat{\mathbf{x}}}(f(\widehat{\mathbf{X}}(t))-f(\widehat{\mathbf{x}})) .
$$

For a function $f$ define its phase function by $\left.\left.f_{\mathbf{p}}: S_{\mathbf{p}}^{-} \cup\right] 0, t^{*}\right]$ $\rightarrow \mathbb{R}^{+}$such that $f_{\mathbf{p}}\left(\mathbf{a}, t_{0}\right)=f(\widehat{\mathbf{x}})$. If for a state $\left(\mathbf{x}, t_{0}\right)$ the function $t \rightarrow f_{\mathbf{p}}\left(\widehat{\phi}_{\mathbf{p}}\left(t,\left(\mathbf{a}, t_{0}\right)\right)\right)$ is differentiable almost everywhere on $\left[0, \widehat{d}_{\text {path }}(\widehat{\mathbf{x}})[\right.$ then, if $\widehat{\phi}_{\mathbf{p}}\left(0,\left(\mathbf{a}, t_{0}\right)\right)=\left(\mathbf{a}, t_{0}\right)$

$$
\frac{\mathrm{d}}{\mathrm{d} t} f_{\mathbf{p}}\left(\widehat{\phi}_{\mathbf{p}}\left(t,\left(\mathbf{a}, t_{0}\right)\right)\right)=\widehat{\mathcal{V}}_{\mathbf{p}} f_{\mathbf{p}}\left(\widehat{\phi}_{\mathbf{p}}\left(t,\left(\mathbf{a}, t_{0}\right)\right)\right)
$$

holds for almost all $t$, and where $\widehat{\mathcal{V}}_{\mathbf{p}}$ is a vector field and $\widehat{\phi}_{\mathbf{p}}\left(0,\left(\mathbf{a}, t_{0}\right)\right)=\left(\mathbf{a}, t_{0}\right)$ is the unique integral curve of $\widehat{\mathcal{V}}_{\mathbf{p}}$ such that (10) is satisfied. Therefore, letting $t$ tend to zero, equation (9) becomes

$$
\widehat{\mathcal{A}} f\left(\mathbf{x}, t_{0}\right)=\widehat{\mathcal{V}} f\left(\mathbf{x}, t_{0}\right)+\boldsymbol{\lambda}(\mathbf{x})\left(\widehat{\mathcal{Q}} f\left(\mathbf{x}, t_{0}\right)-f\left(\mathbf{x}, t_{0}\right)\right)
$$

where with a slight abuse of notation $\widehat{\mathcal{V}} f\left(\mathbf{x}, t_{0}\right)$ should be read as $\widehat{\mathcal{V}} f_{\mathbf{p}}\left(\mathbf{a}, t_{0}\right)$. Finally, we write from now on $t \rightarrow f(\widehat{\phi}(t, \widehat{\mathbf{x}}))$ in place of more cumbersome $t \rightarrow f_{\mathbf{p}}\left(\widehat{\phi}_{\mathbf{p}}\left(t,\left(\mathbf{a}, t_{0}\right)\right)\right)$.

The next result shows that the augmentation of the state space of $\mathbf{X}$ allows to define $U$ as the unique solution of a set of integro-differential equation. The impact of this transformation will become more relevant in Section 4.

Proposition 2. For each $\widehat{\mathbf{x}} \in S_{\widehat{\mathbf{x}}}, t \rightarrow U(\widehat{\phi}(t, \widehat{\mathbf{x}}))$ is absolutely continuous on $\left[0, \widehat{d}_{\text {path }}(\widehat{\mathbf{x}})[\right.$ and $U$ is the unique solution of the equations

$$
\widehat{\mathcal{A}} f(\widehat{\mathbf{x}})=0, \quad \widehat{\mathbf{x}} \in S_{\widehat{\mathbf{x}}},
$$

$f(\Delta)=0$ and at the boundary state $\widehat{\mathbf{x}} \in \widehat{B}, f(\widehat{\mathbf{x}})=\mathbf{I}_{\widehat{B}^{0}}(\widehat{\mathbf{x}})$.

Proof. By definition $U(\Delta)=0$. We assume that $\left.\left.\widehat{\mathbf{x}}=\left(\mathbf{x}, t_{0}\right) \in \mathbf{S}_{\mathbf{X}} \times\right] 0, t^{*}\right]$ and $t \in] 0, \widehat{d}_{\text {path }}(\widehat{\mathbf{x}})[$. By the strong Markov property, we have

$$
\left.U(\widehat{\mathbf{x}})=\widehat{G}_{\widehat{\mathbf{x}}}(t) U(\widehat{\phi}(t, \widehat{\mathbf{x}}))+\mathbb{E}_{\widehat{\mathbf{x}}}\left(\widehat{\mathcal{Q}} U\left(\widehat{\phi}\left(\widehat{T}_{1}, \widehat{\mathbf{x}}\right)\right)\right) \mathbf{I}_{00, t]}\left(\widehat{T}_{1}\right)\right) .
$$


Now, using the density function of first transition $\widehat{T}_{1}$ on $[0, t]$ given by $\lambda(\phi(s, \mathbf{x})) G_{\mathbf{x}}(s)$ and proceeding in a similar way as in proof of Proposition 1, it follows that

$$
\begin{aligned}
U(\widehat{\phi}(t, \widehat{\mathbf{x}}))= & U(\widehat{\mathbf{x}}) \\
& +\int_{0}^{t} \lambda(\phi(s, \mathbf{x}))(U(\widehat{\phi}(s, \widehat{\mathbf{x}}))-\widehat{\mathcal{Q}} U(\widehat{\phi}(s, \widehat{\mathbf{x}}))) \mathrm{d} s
\end{aligned}
$$

Since $\widehat{\mathcal{Q}} U$ is clearly bounded ( $U$ is bounded) the by fundamental theorem of calculus $t \rightarrow U(\widehat{\phi}(t, \widehat{\mathbf{x}}))$ is absolutely continuous on $[0, t]$ and equation (11) is satisfied. Now fix $\widehat{\mathbf{x}}^{\prime} \in \widehat{B}$ and $t>0$ such that the initial state is $\widehat{\mathbf{x}}=\phi\left(-t, \widehat{\mathbf{x}}^{\prime}\right)$. By strong Markov property we have that

$$
\begin{aligned}
U(\widehat{\mathbf{x}})= & \mathbb{E}\left(\mathbf{I}_{\widehat{B}^{0}}\left(\widehat{\mathbf{x}}^{\prime}\right) \mathbf{I}_{\left\{\widehat{T}_{1}=\widehat{d}_{\text {path }}(\widehat{\mathbf{x}})\right\}}+\widehat{\mathcal{Q}} U\left(\widehat{X}\left(\widehat{T}_{1}^{-}\right)\right)\right) \\
= & \mathbb{P}\left(\widehat{T}_{1}=\widehat{d}_{\text {path }}(\widehat{\mathbf{x}})\right)\left(\mathbf{I}_{\widehat{B}^{0}}\left(\widehat{\mathbf{x}}^{\prime}\right)\right. \\
& \left.+\widehat{\mathcal{Q}} U\left(\widehat{\mathbf{x}}^{\prime}\right)\right)+\int_{0}^{t} \lambda(s, \phi(t, \mathbf{x})) G_{\mathbf{x}}(s) \widehat{\mathcal{Q}} U(\widehat{\phi}(s, \widehat{\mathbf{x}})) \mathrm{d} s
\end{aligned}
$$

Letting $t \downarrow 0$ the function $U$ has a limit as a boundary state is approached and $U\left(\widehat{\mathbf{x}}^{\prime}\right)=\mathbf{I}_{\widehat{B}^{0}}\left(\widehat{\mathbf{x}}^{\prime}\right)$.

If $\widehat{\mathbf{x}} \in\left[0, t^{*}\left[\times S_{\widehat{\mathbf{x}}}, t \rightarrow f(\widehat{\phi}(t, \widehat{\mathbf{x}}))\right.\right.$ is an absolutely continuous function on $[0, \widehat{d}(\widehat{\mathbf{x}})[$ and $f$ is bounded, from conditions from [Davis [3], Theorem 31.3, p. 83] conveniently applied, the process $\left(\widehat{M}^{f}(t)\right)$ defined by

$$
\begin{aligned}
\widehat{M}^{f}(t) & =f(\widehat{\mathbf{X}}(t))-f(\widehat{\mathbf{X}}(0)) \\
& -\sum_{\left\{\widehat{T}_{i} \leq t, \widehat{\mathbf{X}}\left(\widehat{T}_{i}^{-}\right) \in \widehat{B}\right\}}\left(\widehat{\mathcal{Q}} f\left(\widehat{\mathbf{X}}\left(\widehat{T}_{i}\right)\right)-f\left(\widehat{\mathbf{X}}\left(\widehat{T}_{i}^{-}\right)\right)\right), \quad t \geq 0
\end{aligned}
$$

is a martingale. Taking conditional expectations, $\mathbb{E}_{\widehat{\mathbf{x}}}\left(\widehat{M}^{f}(t)\right)$ $=\widehat{M}^{f}(0)=0$ and therefore

$$
\begin{aligned}
f(\widehat{\mathbf{x}})= & \mathbb{E}_{\widehat{\mathbf{x}}} f(\widehat{\mathbf{X}}(t)) \\
& -\mathbb{E}_{\widehat{\mathbf{X}}}\left(\sum_{\left\{\widehat{T}_{i} \leq t, \widehat{\mathbf{X}}\left(\widehat{T}_{i}^{-}\right) \in \widehat{B}\right\}}\left(\widehat{\mathcal{Q}} f\left(\widehat{\mathbf{X}}\left(\widehat{T}_{i}\right)\right)-f\left(\widehat{\mathbf{X}}\left(\widehat{T}_{i}^{-}\right)\right)\right)\right) .
\end{aligned}
$$

In view of $f(\widehat{\mathbf{x}})=\mathbf{I}_{\widehat{B}^{0}}(\widehat{\mathbf{x}})$ and $\widehat{\mathcal{Q}} f(\widehat{\mathbf{x}})=0$ for $\widehat{\mathbf{x}} \in \widehat{B}$, we have that $\widehat{\mathcal{Q}} f\left(\widehat{\mathbf{X}}\left(\widehat{T}_{i}\right)\right)-$ $f\left(\widehat{\mathbf{X}}\left(\widehat{T}_{i}^{-}\right)\right)=-\mathbf{I}_{\widehat{B}^{0}}\left(\widehat{T}_{i}^{-}\right)$. Since $\widehat{\mathbf{X}}(t)=\Delta$ for all $t \geq t^{*}, \mathbb{E}_{\widehat{\mathbf{x}}} f(\widehat{\mathbf{X}}(t))=0$ as $t$ tends to infinity. Letting $t \rightarrow \infty$ the solution of the equation (11) is unique and $f=U$. 
The mean path duration and the path persistence written as expectations of functionals of the multihop path process are the unique solutions of a system of integro-differential equations. However, any direct method to solve them is quite problematic and depends very much on the specific characterization of the multihop path process (number of nodes, deterministic motion, jump rate, transition measure).

\section{Recursive computations}

In this section we give a recursive scheme which provides the basis for practical numerical techniques for computing the path metrics defined in Section 3.

\subsection{Mean path duration}

Let $D^{0}$ be a function such that $D^{0}(\mathbf{x})=0$ for all $\mathbf{x} \in S_{\mathbf{x}}^{\Delta}$ and let $\mathcal{O}$ be an operator mapping the set of bounded measurable functions on $S_{\mathbf{x}}^{\Delta} \cup B$ into itself. The action of the operator $\mathcal{O}$ on $D^{0}$ gives the function $D^{1} \equiv \mathcal{O} D^{0}$ defined by

$$
D^{1}(\mathbf{x})=\mathbb{E}_{\mathbf{x}}\left(\int_{0}^{T_{1}} \mathbf{I}_{S \mathbf{X}}(X(s)) \mathrm{d} s+D^{0}\left(\mathbf{X}\left(T_{1}\right)\right)\right), \mathbf{x} \in S_{\mathbf{X}}^{\Delta} .
$$

Iterating $k(\geq 1)$ times the operator $\mathcal{O}$ on $D^{0}$ results into the function $D^{k} \equiv$ $\mathcal{O}^{k} D^{0}$ given by

$$
\begin{aligned}
D^{k}(\mathbf{x}) & =\mathcal{O} D^{k-1}(\mathbf{x}) \\
& =\mathbb{E}_{\mathbf{x}}\left(\int_{0}^{T_{1}} \mathbf{I}_{S_{\mathbf{X}}}(X(s)) \mathrm{d} s+D^{k-1}\left(\mathbf{X}\left(T_{1}\right)\right)\right)
\end{aligned}
$$

for $\mathbf{x} \in S_{\mathbf{x}}^{\Delta}$. The metric $D^{k}(\mathbf{x})$ denotes the mean path duration constrained to at most $k$ jumps of the multihop process $\mathbf{X}$ starting from state $\mathbf{x}$. As the number of jumps increases we obtain in the limit the mean path duration $D(\mathbf{x})$ defined by (3).

Proposition 3. For each $\mathbf{x} \in \mathbf{S}_{\mathbf{X}}$ and $k \geq 1$,

$$
D^{k}(\mathbf{x})=\mathbb{E}_{\mathbf{x}}\left(\int_{0}^{T_{k}} \mathbf{I}_{S_{\mathbf{X}}}(X(s)) \mathrm{d} s+D^{0}\left(\mathbf{X}\left(T_{k}\right)\right)\right)
$$

and 


$$
\lim _{k \rightarrow \infty} D^{k}(\mathbf{x})=D(\mathbf{x})
$$

Proof. For $k=1$, equation (12) follows from definition. Suppose that (12) holds for a fixed $k$ then

$$
\begin{aligned}
D^{k+1}(\mathbf{x}) & =\mathcal{O}^{k}\left(\mathcal{O} D^{0}\right)(\mathbf{x}) \\
& =\mathbb{E}_{\mathbf{x}}\left(\int_{0}^{T_{k}} \mathbf{I}_{S_{\mathbf{X}}}(X(s)) \mathrm{d} s+D^{1}\left(\mathbf{X}\left(T_{k}\right)\right)\right) .
\end{aligned}
$$

By the strong Markov property,

$$
\begin{aligned}
D^{k+1}(\mathbf{x}) & =\mathbb{E}_{\mathbf{x}}\left(\int_{0}^{T_{k}} \mathbf{I}_{S_{\mathbf{X}}}(X(s)) \mathrm{d} s\right) \\
& +\mathbb{E}_{\mathbf{X}}\left(\int_{T_{k}}^{T_{k+1}} \mathbf{I}_{S_{\mathbf{X}}}(X(s)) \mathrm{d} s+D^{0}\left(T_{k+1}\right) \mid \mathcal{F}_{T_{k}}\right) \\
& =\mathbb{E}_{\mathbf{x}}\left(\int_{0}^{T_{k+1}} \mathbf{I}_{S_{\mathbf{X}}}(X(s)) \mathrm{d} s+D^{0}\left(T_{k+1}\right)\right) .
\end{aligned}
$$

where $\mathcal{F}_{T_{k}}$ is the history of $\mathbf{X}$ until time $T_{k}$. Since $T_{k} \rightarrow \infty$ w.p. 1 as $k \rightarrow \infty$ for all initial states $\mathbf{x} \in \mathbf{S}_{\mathbf{X}}$, equation (13) follows by monotone convergence.

From the definition of $D^{k}$ and proceeding as in (4), for $\mathbf{x} \in S_{\mathbf{X}}$ we obtain

$$
\mathcal{A} D^{k}(\mathbf{x})=\mathcal{V} D^{k}(\mathbf{x})+\boldsymbol{\lambda}(\mathbf{x})\left(\mathcal{Q} D^{k-1}(\mathbf{x})-D^{k}(\mathbf{x})\right)
$$

where $\mathcal{Q}$ now acts on the function $D^{k-1}$. To establish the next result, we use Proposition 32.20 in [3] conveniently adapted to the PDMP X.

Proposition 4. Suppose that the function $D^{k-1}$ is given. For each $\mathbf{x} \in S_{\mathbf{X}}$, $t \rightarrow D^{k}(\phi(t, \mathbf{x}))$ is absolutely continuous function on $\left[0, d(\mathbf{x})\left[\right.\right.$ and $D^{k}$ is the unique bounded solution of the equations

$$
\mathcal{V} f(\mathbf{x})+\boldsymbol{\lambda}(\mathbf{x})\left(\mathcal{Q} D^{k-1}(\mathbf{x})-f(\mathbf{x})\right)=-1, \quad \mathbf{x} \in \mathbf{S}_{\mathbf{X}},
$$

and at a boundary state $\mathbf{x} \in B, f(\mathbf{x})=f(\Delta)=0$.

The difference between equations (14) and (6) is that now the operator $\mathcal{Q}$ acts only on the given function $D^{k-1}$ which turn it in ODE. Combining this result with Proposition 3 provides a recursive way of computing the mean path duration $D$.

Proof. To show that $D^{k}$ satisfies equation (14) and $t \rightarrow D^{k}(\phi(t, \mathbf{x}))$ is absolutely continuous we may follow along the same lines as in the proof of Proposition 1 with the difference that the operator $\mathcal{Q}$ now acts on the given 
function $D^{k-1}$. The same also holds to prove that $D^{k}$ satisfies the boundary condition.

Fix a state $\mathbf{x} \in \mathbf{S}_{\mathbf{X}}$ such that $d_{\text {path }}(\mathbf{x})=\infty$. If we write $y_{\mathbf{x}}(t)=f(\phi(t, \mathbf{x}))$, $a_{\mathbf{x}}(t)=\boldsymbol{\lambda}(\phi(t, \mathbf{x}))$ and $b_{\mathbf{x}}(t)=1+\boldsymbol{\lambda}(\phi(t, \mathbf{x})) \mathcal{Q} D^{k-1}(\phi(t, \mathbf{x}))$, then equation (14) is a linear scalar ODE with general coefficients

$$
\frac{\mathrm{d}}{\mathrm{d} t} y_{\mathbf{x}}(t)=a_{\mathbf{x}}(t) y_{\mathbf{x}}(t)-b_{\mathbf{x}}(t), \quad y_{\mathbf{x}}(0)=f(\mathbf{x})
$$

which has a unique solution along $\left\{\phi(t, \mathbf{x}), t<d_{\text {path }}(\mathbf{x})\right\}$ given by

$$
\begin{aligned}
y_{\mathbf{x}}(t)= & \exp \left(\int_{0}^{t} a_{\mathbf{x}}(s) \mathrm{d} s\right) f(\mathbf{x}) \\
& -\int_{0}^{t} \exp \left(\int_{s}^{t} a_{\mathbf{x}}(u) \mathrm{d} u\right) b_{\mathbf{x}}(s) \mathrm{d} s .
\end{aligned}
$$

Using the distribution of $T_{1}, D^{k}(\mathbf{x})$ can be expressed as

$$
D^{k}(\mathbf{x})=\int_{0}^{\infty} \exp \left(\int_{0}^{s}-a_{\mathbf{x}}(u) \mathrm{d} u\right) b_{\mathbf{x}}(s) \mathrm{d} s .
$$

Since $b_{\mathbf{x}}(t)$ is bounded and $a_{\mathbf{x}}(t)>0$, direct calculations shows that the unique solution for which $y_{\mathbf{x}}(t)$ is bounded is given by the initial condition $y_{\mathbf{x}}(0)=f(\mathbf{x})=D^{k}(\mathbf{x})$ and therefore

$$
y_{\mathbf{x}}(t)=\int_{t}^{\infty} \exp \left(\int_{t}^{s}-a_{\mathbf{x}}(u) \mathrm{d} u\right) b_{\mathbf{x}}(s) \mathrm{d} s
$$

with $y_{\mathbf{x}}(t)=f(\phi(t, \mathbf{x}))=D^{k}(\phi(t, \mathbf{x}))$.

Suppose now that $\mathbf{x} \in \mathbf{S}_{\mathbf{X}}$ such that $d_{\text {path }}(\mathbf{x})<\infty$. Let $\mathbf{x}^{\prime}=\phi\left(d_{\text {path }}(\mathbf{x}), \mathbf{x}\right)$ and $t \in\left[-d_{\text {path }}(\mathbf{x}), 0\right]$, then equation (15) becomes

$$
\frac{\mathrm{d}}{\mathrm{d} t} y_{\mathbf{x}^{\prime}}(t)=a_{\mathbf{x}^{\prime}}(t) y_{\mathbf{x}^{\prime}}(t)-b_{\mathbf{x}^{\prime}}(t), \quad y_{\mathbf{x}^{\prime}}(0)=f\left(\mathbf{x}^{\prime}\right)
$$

since the solution of the equation is unique and $f\left(\mathbf{x}^{\prime}\right)=D^{k}\left(\mathbf{x}^{\prime}\right)=0$ by the boundary condition, then

$$
y_{\mathbf{x}^{\prime}}(t)=f\left(\phi\left(t, \mathbf{x}^{\prime}\right)\right)=D^{k}\left(\phi\left(t, \mathbf{x}^{\prime}\right)\right) .
$$

This completes the proof. 


\subsection{Path persistence}

Define the function $U^{0}$ such that $U^{0}(\widehat{\mathbf{x}})=0$ for all $\widehat{\mathbf{x}} \in S_{\widehat{\mathbf{x}}}^{\Delta}$. Let $\widehat{\mathcal{O}}$ denote an operator acting on the domain of bounded measurable real valued functions on $\mathbf{S}_{\widehat{\mathbf{X}}}^{\Delta} \cup \widehat{B}$ such that the operation of $\widehat{\mathcal{O}}$ on $U^{0}$ gives the function $U^{1} \equiv \widehat{\mathcal{O}} U^{0}$ given by

$$
U^{1}(\widehat{\mathbf{x}})=\mathbb{E}_{\widehat{\mathbf{x}}}\left(\mathbf{I}_{\widehat{B}^{0}}\left(\widehat{\mathbf{X}}\left(\widehat{T}_{1}^{-}\right)\right)+U^{0}\left(\widehat{\mathbf{X}}\left(\widehat{T}_{1}\right)\right)\right), \quad \widehat{\mathbf{x}} \in S_{\widehat{\mathbf{X}}}^{\Delta}
$$

Iterating successively $k(>1)$ times the operator $\widehat{\mathcal{O}}$ on $U^{0}$ results into the function $U^{k} \equiv \widehat{\mathcal{O}}^{k} U^{0}$ given by

$$
\left.U^{k}(\widehat{\mathbf{x}})=\widehat{\mathcal{O}} U^{k-1}(\widehat{\mathbf{x}})=\mathbb{E}_{\widehat{\mathbf{x}}}\left(\mathbf{I}_{\widehat{B}^{0}} \widehat{\mathbf{X}}\left(\widehat{T}_{1}^{-}\right)\right)+U^{k-1}\left(\widehat{\mathbf{X}}\left(\widehat{T}_{1}\right)\right)\right)
$$

for $\widehat{\mathbf{x}} \in S_{\widehat{\mathbf{x}}}^{\Delta}$. The metric $U^{k}\left(\mathbf{x}, t_{0}\right)$ for $\left(\mathbf{x}, t_{0}\right) \in S_{\widehat{\mathbf{x}}}$ denotes the path persistence at time $t_{0}$ constrained to at most $k$ jumps of the process $\widehat{\mathbf{X}}$ starting from state $\left(\mathbf{x}, t_{0}\right)$. As $k$ tends to infinity, we obtain the expectation functional $U(\widehat{\mathbf{x}})$. By the strong Markov property and the definition of $\widehat{\mathbf{X}}$, we can state the following result.

Proposition 5. For each $\widehat{\mathbf{x}} \in S_{\widehat{\mathbf{x}}}$ and $k \geq 1$,

$$
U^{k}(\widehat{\mathbf{x}})=\mathbb{E}_{\widehat{\mathbf{x}}}\left(\sum_{i=1}^{k} \mathbf{I}_{\widehat{B}^{0}}\left(\widehat{\mathbf{X}}\left(\widehat{T}_{i}^{-}\right)\right)+U^{0}\left(\widehat{\mathbf{X}}\left(\widehat{T}_{k}\right)\right)\right)
$$

and

$$
\lim _{k \rightarrow \infty} U^{k}(\widehat{\mathbf{x}})=U(\widehat{\mathbf{x}}) .
$$

Using the definition of $U^{k}$ and following the same steps as in (9), we have, for $\left(\mathbf{x}, t_{0}\right) \in S_{\widehat{\mathbf{x}}}$

$$
\widehat{\mathcal{A}} U^{k}\left(t_{0}, \mathbf{x}\right)=\widehat{\mathcal{V}} U^{k}\left(\mathbf{x}, t_{0}\right)+\boldsymbol{\lambda}(\mathbf{x})\left(\widehat{\mathcal{Q}} U^{k-1}\left(\mathbf{x}, t_{0}\right)-U\left(\mathbf{x}, t_{0}\right)\right),
$$

where now $\widehat{\mathcal{Q}}$ acts on the function $U^{k-1}$. This gives rise to the following result whose proof is omitted, since it uses merely the arguments used in the proof of Proposition 4.

Proposition 6. Suppose that the function $U^{k-1}$ is given. Then for each $\widehat{\mathbf{x}} \in$ $S_{\widehat{\mathbf{x}}}, t \rightarrow U^{k}(\widehat{\phi}(t, \widehat{\mathbf{x}}))$ is absolutely continuous function on $\left[0, \widehat{d}_{\text {path }}(\widehat{\mathbf{x}})\left[\right.\right.$ and $U^{k}$ satisfies and is the unique solution of the equations

$$
\widehat{\mathcal{V}} f\left(\mathbf{x}, t_{0}\right)+\boldsymbol{\lambda}(\mathbf{x})\left(\widehat{\mathcal{Q}} U^{k-1}\left(t_{0}, \mathbf{x}\right)-f\left(\mathbf{x}, t_{0}\right)\right)=0
$$

for $\left(\mathbf{x}, t_{0}\right) \in S_{\widehat{\mathbf{X}}}, f(\Delta)=0$ and, at a boundary state $\widehat{\mathbf{x}} \in \widehat{B}, f(\widehat{\mathbf{x}})=\mathbf{I}_{\widehat{B}^{0}}(\widehat{\mathbf{x}})$. 
Computing $D^{k}$ (resp. $U^{k}$ ) requires only to solve ODEs instead of solving integro-differential equations. The results of these calculations are then used to compute the next iteration $k+1$. Since they are independent ODEs they can be computed using parallel computation. The convergence of the solution depends on how large $k$ has to be before $D^{k}$ (resp. $U^{k}$ ) is close to $D$ (resp. $U)$. The derivation of error bounds for these quantities is possible, as shown in the Appendix, which also gives an estimate of number of iterations needed for convergence.

Any direct implementation of these equations requires a discretization of the state space and solving at each grid point an independent ODE, providing the data for calculating the next iteration. Therefore, numerical software routines that implement the corresponding differential equations in a recursive way are needed for obtaining the final results. It is unrealistic to hope that numerical solutions are possible for a medium size number of links in a single workstation due to the great number of computer processing cycles and the need of storing large amounts of data. However, it is possible to solve the equations in the case of one or two links and in one dimensional ad hoc networks. It should be noted that, for numerical problems of this kind, the use of distributed computing for studying the reliability of multihops paths in a general scenario seems to be a conditio sine qua non. Such a development will be efficient if, in addition, we use techniques such as function interpolation and eliminate sample paths whose contribution is negligible. It seems that the equations presented in this section can be effectively solved for non trivial cases, however, more research in this direction is necessary.

\section{Numerical Results}

In this section we illustrate an application of the preceding results to study the differences between independent links. The scenario proposed is based on [6] with pause times and restricted mobility direction of nodes, which intends to model a military scenario where vehicles move in low velocities in a given direction. We consider that the phase durations are exponentially distributed with means of $30 \mathrm{~s}$ and $120 \mathrm{~s}$ in move and pause phase, respectively. The transmission range of a node is set up to $250 \mathrm{~m}$. The mobility vector is obtained choosing a velocity $(\mathrm{m} / \mathrm{s})$ and direction of nodes uniformly distributed in $] 10,20[$ and $]-\pi / 4, \pi / 4[$, respectively. For a multihop path with $N$ nodes, initially each node $i(2 \leq i \leq N)$ is deployed inside node $i-1$ 's radio coverage with an angle uniformly distributed in $]-\pi / 4, \pi / 4$ [ and with a distance following a triangular distribution in the interval $(0,250)$ and mode 62.5 . The initial phase of a node is picked randomly with probabilities proportional to the mean time spent in the phase. If the initial phase is move, the mobility vector is chosen as if at a transition phase. 


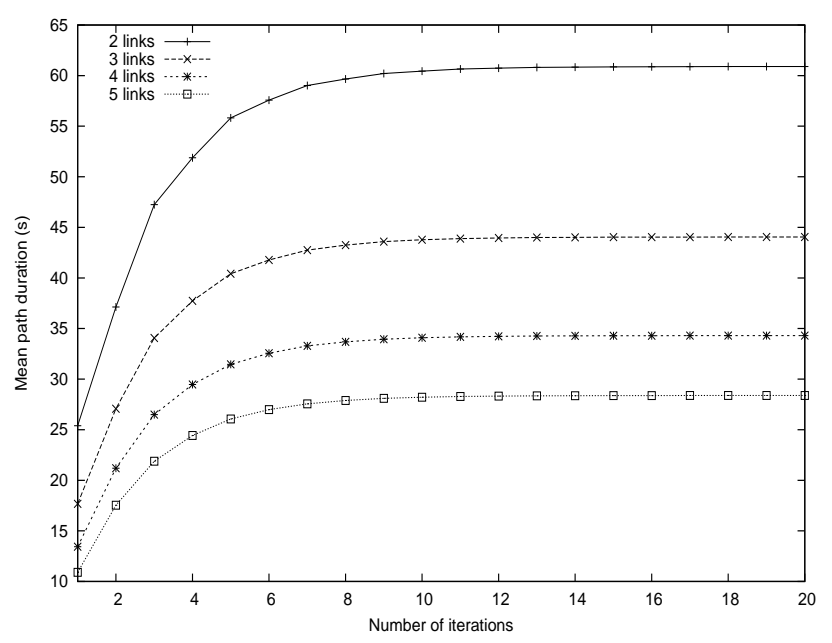

Fig. 1 Mean path duration after each iteration.

Figure 1 shows the results of the mean path duration after each iteration for different link count. The departure states of the multihop path were sampled according to the initial distribution and their respective mean path durations were estimated in each iteration using Monte Carlo methods (in a single computer workstation). The results were averaged out in the final of each iteration. The difference between iterations gets smaller as the number of links increases since it is more likely that a path failure occurs after a small number of phase transitions. However, all curves have converged before iteration 20 .

In Figure 2 we investigate the impact of neglecting the dependency between links in the mean path duration for different link count. Numerical routines were developed for independent links. Since each link is only dependent on its neighboor links the difference between mean path durations reaches its maximum value for two links and decreases with the increase of link count. The percentage error from assuming independent links is always higher than $15 \%$.

Figure 3 depicts the path persistence for 2 and 5 -links in the interval $[0,200]$. The initial states of the multihop path were selected according to the initial distribution (described above) and the paths persistence were estimated in each iteration through Monte Carlo methods. After convergence, the results were averaged out. The curves obtained have been plotted against the independent link assumption. The marginal probabilities of independent links were computed using the results of PDMP with one link. For 2-link paths the maximum difference between the curves is smaller than for 5-link paths but persists for a longer time since the path duration is stochastically decreasing in the number of links. As expected, for values of $t_{0}$ at the be- 


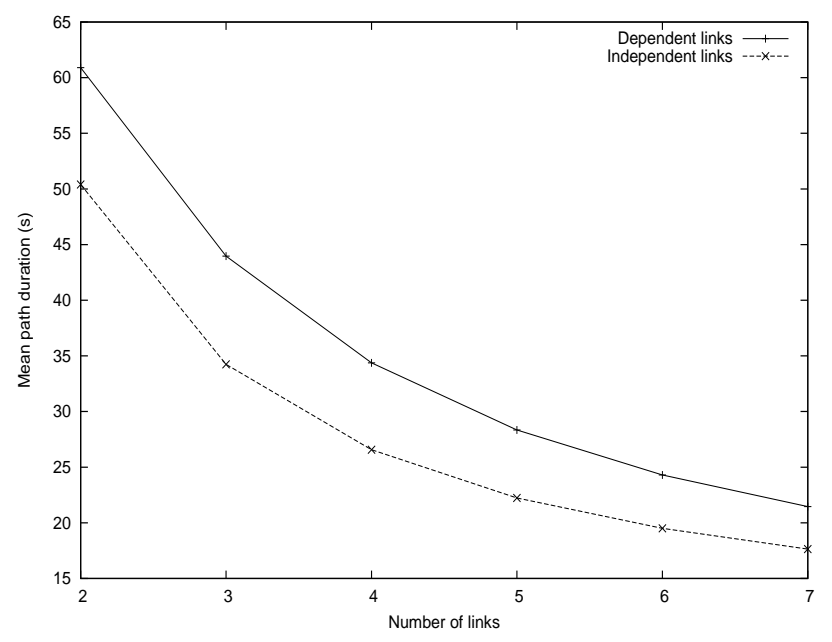

Fig. 2 Mean path duration $v s$ link count.

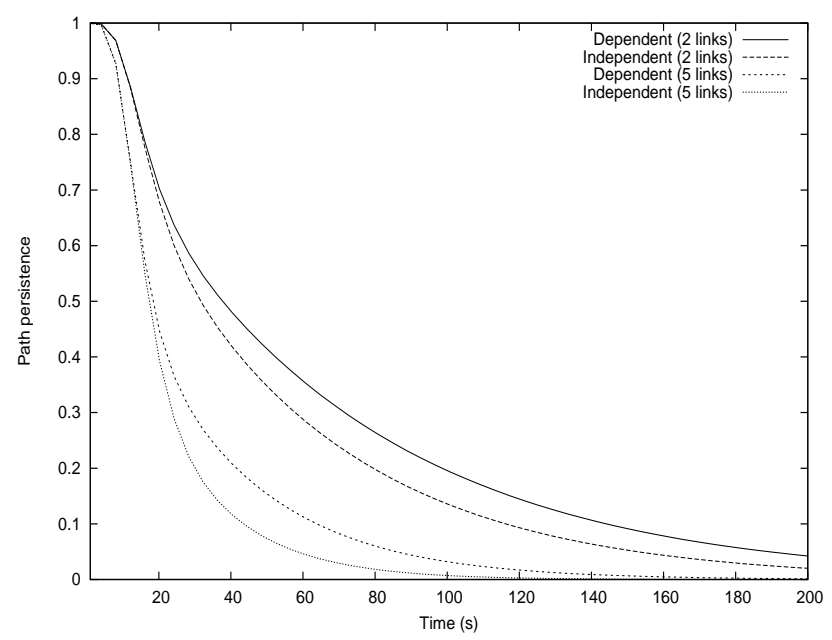

Fig. 3 Path persistence vs link count.

ginning and at the end of the interval the differences are small. Finally, the independent link failure assumption leads to underestimation of the path metrics in the scenario presented.

In Figure 4 we study the impact of mean time in pause phase on the mean duration of a 4-link path. The results are rather sensitive to the mean value of pause phase and getting an estimate for the mean path duration using the link independence assumption may in fact be a major problem when the inactive time of a node is large. 


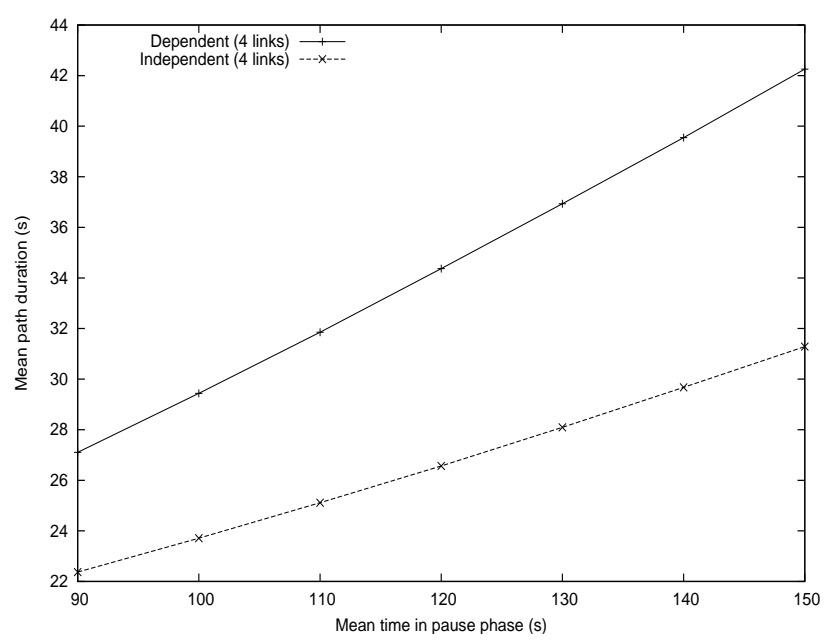

Fig. 4 Mean path duration vs mean pause phase.

In spite of the goal of this paper being to present an analytical framework to study path reliability, we highlight from our experiments that slower moving nodes along with shorter link distances conducts to more significant differences in the path metrics when compared with the corresponding independent link approximations.

\section{Conclusion}

This paper developed an analytical framework to fully characterize the random behavior of a multihop path under a PDMP. The proposed framework enabled to describe the mean path duration and the path persistence through a set of integro-differential equations. Further, we have presented a recursive scheme to compute these path metrics. Numerical results show the adequacy of the framework developed to obtain the path metrics which were compared with the corresponding independent link approximations. These results enabled to accurately determine the reliability of communications paths and can be employed to improve the performance of routing algorithms in MANETs. Future work will include the use of other individual (or group) mobility models of nodes [2] that admit a PDMP description of a multihop path; the application of our analytical framework to derive other path metrics; and distributed implementation of the recursive equations. 


\section{Appendix}

We prove here a result which was used in the proof of Proposition 1.

Proposition 7. The expected path duration $D(\mathbf{x})$ is a bounded function of $\mathbf{x} \in \mathbf{S}_{\mathbf{X}}^{\Delta}$ and $\mathbb{E}_{\mathbf{x}}(D(\mathbf{X}(t)))$ goes to zero as $t$ tends to infinity.

Proof (Proposition 1). For any multihop path process $(\mathbf{X}(t))$ with initial state $\mathbf{x}=(\mathbf{p}, \mathbf{a}) \in \mathbf{S}_{\mathbf{X}}$, we have that

$$
D(\mathbf{x}) \leq D\left(\mathbf{x}^{\prime}\right)
$$

where $D\left(\mathbf{x}^{\prime}\right)$ denotes the expected path duration of a multihop path process $\left(\mathbf{X}^{\prime}(t)\right)$ with one hop whose initial state

$$
\left.\mathbf{x}^{\prime}=\left(\mathbf{p}^{\prime}, \mathbf{a}^{\prime}\right)\right), \mathbf{p}^{\prime}=\left(p^{j}, p^{j+1}\right)
$$

and

$$
\mathbf{a}^{\prime}=\left(\left(e^{j}, e^{j+1}\right),\left(m^{i}, m^{j+1}\right), l_{r}^{j+1}\right),
$$

$j=1, \ldots, n-1$ is given by the state of two consecutive nodes of $(\mathbf{X}(t))$ at time 0 .

If $T_{i}^{\prime}, i \geq 1$ are the jump times of the process $\left(\mathbf{X}^{\prime}(t)\right)$ then taking $T_{0}^{\prime}=0$ and $S_{i}^{\prime}=T_{i+1}^{\prime}-T_{i}^{\prime}$, we can write

$$
D\left(\mathbf{x}^{\prime}\right)=\mathbb{E}_{\mathbf{x}^{\prime}}\left(\sum_{i=0}^{\infty} S_{i}^{\prime} \mathbf{I}_{S_{\mathbf{X}}}\left(X^{\prime}\left(T_{i}^{\prime}\right)\right)\right) .
$$

Given that at a jump time the path is not broken, the expected time between jumps is bounded by

$$
\mathbb{E}_{\mathbf{x}^{\prime}}\left(S_{i}^{\prime} \mathbf{I}_{S_{\mathbf{X}}}\left(X^{\prime}\left(T_{i}^{\prime}\right)\right)\right) \leq K \equiv 1 /\left(2 \min \left(\lambda_{0}^{\min }, \lambda_{1}^{\min }\right)\right)
$$

where we recall that we assume that hazard rate functions of the times distributions in the phases are bounded such that $0<\lambda_{i}^{\min } \leq \lambda_{i}(t) \leq \lambda_{i}^{\max }$. If at time $T_{i}^{\prime}$ the process is in a state $\mathbf{X}^{\prime}\left(T_{i}^{\prime}\right)$ where the two nodes are in different phases then, $d_{\text {path }}\left(\mathbf{X}^{\prime}\left(T_{i}^{\prime}\right)\right)$, the duration of the path constrained to no phase transitions of the nodes, is smaller or equal to $2 R / v_{\min }$ (i.e. the maximum time it takes for a node in move phase to cross the transmission range of a node in pause phase). Hence,

$$
\begin{aligned}
& \mathbb{E}_{\mathbf{x}^{\prime}}\left(\mathbf{I}_{S_{\mathbf{X}}}\left(X^{\prime}\left(T_{i}^{\prime}\right)\right) \mid \mathbf{X}^{\prime}\left(T_{i}^{\prime}\right) \in S_{(0,1)}^{-} \cup S_{(1,0)}^{-}\right) \\
& \quad \leq p \equiv 1-\exp \left(-2 R / v_{\min }\left(\lambda_{0}^{\max }+\lambda_{1}^{\max }\right)\right)
\end{aligned}
$$

Note that for a multihop path process $\left(\mathbf{X}^{\prime}(t)\right)$ with two nodes the jumps alternate between a state where both nodes are in different phases or in the 
same phase. Therefore, assuming that the path just breaks when the two nodes are in different phases, the probability that the path is alive after $i$ jumps is bounded by

$$
\mathbb{E}_{\mathbf{x}^{\prime}}\left(\mathbf{I}_{S_{\mathbf{X}}}\left(X^{\prime}\left(T_{i}^{\prime}\right)\right)\right) \leq p^{\lfloor i / 2\rfloor}
$$

where $\lfloor i / 2\rfloor$ is the minimum number of jumps from a state where the two nodes are in different phases in $i$ jumps of the process from any initial state $\mathbf{x}^{\prime}$. Thus, putting all together, we have that

$$
D\left(\mathbf{x}^{\prime}\right) \leq K \sum_{i=1}^{\infty} p^{\lfloor i / 2\rfloor}=\frac{K(1+p)}{1-p}
$$

which proves the first statement of the proposition. From the above

$$
\begin{aligned}
\mathbb{E}_{\mathbf{x}}(D(\mathbf{X}(t))) & =\mathbb{E}_{\mathbf{x}}\left(D(\mathbf{X}(t)) \mathbf{I}_{S_{\mathbf{X}}}\left(X^{\prime}(t)\right)\right) \\
& \leq D\left(\mathbf{x}^{\prime}\right) \mathbb{P}\left(X^{\prime}(t) \in S_{\mathbf{X}}\right) .
\end{aligned}
$$

Since $\lim _{t \rightarrow \infty} \mathbb{P}\left(X^{\prime}(t) \in S_{\mathbf{X}}\right)=0$, the Proposition is proved.

\section{References}

1. F. Bai, N. Sadagopan, B. Krishnamachari, and A. Helmy. Modeling path duration distributions in MANETs and their impact on reactive routing protocols. IEEE J. Select. Areas Commun., 22(7):1357-1373, 2004.

2. T. Camp, J. Boleng, and V. Davies. A survey of mobility models for ad hoc network research. Wireless Communications and Mobile Computing, 2(5):483502, 2002.

3. M. Davis. Markov Models and Optimization. Chapman and Hall, London/New York, 1993.

4. Y. Han, R. J. La, A. M. Makowski, and S. Lee. Distribution of path durations in mobile ad-hoc networks: Palm's theorem to the rescue. Computer Networks, 50(12):1887-1900, 2006.

5. D. Hong and S. Rappaport. Traffic model and performance analysis for cellular mobile radio telephone systems with prioritized and nonprioritized handoff procedures. IEEE Trans. Veh. Technol., 35(3):77-92, 1986.

6. R. J. La and Y. Han. Distribution of path durations in mobile ad hoc networks and path selection. IEEE/ACM Trans. Netw., 15(5):993-1006, 2007.

7. A. B. McDonald and T. Znati. A path availability model for wireless ad-hoc networks. In Proc. IEEE Wirel. Commun. Netw. Conf., volume 1, pages 35-40, Orleans, LA, USA., 1999.

8. P. Samar and S. Wicker. On the behavior of communications links of a node in a multi-hop mobile environment. In Proc. 5th ACM Int. Symp. Mobile Ad Hoc Netw. Comput., pages 145-156, Tokyo, Japan, 2004.

9. S. Xu, K. L. Blackmore, and H. M. Jones. An analysis framework for mobility metrics in mobile ad hoc networks. EURASIP J. Wirel. Commun. and Network., 1(16):26-26, 2007. 